\title{
An Improved Pseudolite Positioning Accuracy Model Based on Centroid Algorithm
}

\author{
Lian Meng ${ }^{1, a}$, *Sun $\mathrm{Yi}^{2, \mathrm{~b}}$ \\ ${ }^{1,2}$ Beijing University of Posts and Telecommunications, 100876, China \\ a mail: lianmeng@bupt.edu.cn, *mail: sunyiisse@bupt.edu.cn
}

Keywords: pseudolite, centroid algorithm, pseudorange difference, parity correlation coefficient algorithm, phase smooth pseudorange differential measurement

\begin{abstract}
GPS positioning is increasingly used in various fields. However, the cost of GPS satellites is extremely high and the atmosphere affects the output a lot, so it is not suitable for small-scale precise positioning (less than $100 \mathrm{~km}$ ). Aiming at this problem, this paper proposes an enhanced precision model of pseudolite positioning. Based on the idea of pseudolites combined with the centroid algorithm, this model improves the pseudorange accuracy by pseudorange difference, parity correlation coefficient algorithm and phase smooth pseudorange differential measurement. Through the simulation experiment, the improved model can effectively improve the positioning accuracy and reduce the error.
\end{abstract}

\section{Introduction:}

A pseudolite is a quasi-"navigation satellite" that is set up near the ground and has the same effect as a navigation satellite. The function and positioning principle of pseudolite are basically the same with navigation satellites. Because the base station set on the ground, the cost of pseudolite is low and the precise positioning of the base station is also easier to get. However, the geometrical structure of the pseudolite base station has great influence on its positioning progress, which also limits the range of pseudolites. Therefore, this paper presented a new approach that combined with features of the low cost of pseudolite and centroid algorithm through the placement of a large number of pseudolite base stations and this approach can greatly improve the positioning accuracy.

\section{An Improved Pseudolite Positioning Accuracy Improvement Model Based on Centroid} Algorithm:

Because of the curvature of the Earth, the scope of the role of Pseudolite is limited and the general range is $100 \mathrm{~km}$ or less. Thus, the pseudorange difference [1] can be used to correct the output pseudorange and greatly reduce the computational complexity. 
In the range of pseudolites, there exists a known base station and the pseudorange $\mathrm{P}$ its reference receiver receiver measures to the jth pseudolite is:

$P_{r}^{j}=\rho_{r t}^{j}+C\left(d t^{j}-d T_{r r}\right)+d \rho_{r}^{j}+d_{\text {rion }}^{j}+d_{r t r o p}^{j}$

$P_{r}^{j}$ is the pseudorange of the reference receiver to the jth pseudolite at time $\mathrm{t}$; the known amount $\rho_{r t}^{j}$ is the true distance from the reference receiver to the jth pseudolite; $d t^{j}$ is the deviation of the jth pseudo- satellite clock to the GPS time system; $d \rho_{r}^{j}$ is deviation of the reference receiver to the GPS time-series system; $d \rho_{r}^{j}$ is the distance error caused by the $\mathrm{p}$ pseudolite ephemeris at the reference receiver; ${ }^{d}{ }_{\text {rion }}^{j}$ is the error due to the ionospheric delay at the reference receiver; ${ }_{r \text { rrop }}^{j}$ is the error in the reference receiver for tropospheric delay; $\mathrm{C}$ si the speed of light.

The pseudorange correction value for the base station is:

$\Delta \rho_{r}^{j}=\rho_{r t}^{j}-P_{r}^{j}=-C\left(d t^{j}-d T_{r r}\right)-d \rho_{r}^{j}-d_{\text {rion }}^{j}-d_{r t r o p}^{j}$

Similarly, for an unknown dynamic receiver $\mathrm{k}$ in the same range of motion carriers,

the pseudorange of the jth pseudolite is : $P_{k}^{j}=\rho_{k t}^{j}+C\left(d t^{j}-d T_{k r}\right)+d \rho_{k}^{j}+d_{k i o n}^{j}+d_{k \text { trop }}^{j}$

According to the differential GPS principle, the satellite ephemeris error, the ionospheric error, and the tropospheric delay have the consistency of the spatial and temporal characteristics and the pseudorange modification values can be substituted into the above equation the satellite clock error. $P_{k}^{j}+\Delta \rho_{r}^{j}=\rho_{k t}^{j}+C\left(d T_{r r}-d T_{k r}\right)+\left(d \rho_{k}^{j}-d \rho_{r}^{j}\right)+\left(d_{\text {kion }}^{j}-d_{\text {rion }}^{j}\right)+\left(d_{k \text { trop }}^{j}-d_{\text {troop }}^{j}\right)$

Since the distance between stations is less than $100 \mathrm{~km}$, it can be approximated :

$$
\begin{aligned}
& d \rho_{k}^{j}=d \rho_{r}^{j} ; d_{\text {kion }}^{j}=d_{\text {rion }}^{j} ; d_{\text {ktrop }}^{j}=d_{r t r o p}^{j} \\
& P_{k}^{j}+\Delta \rho_{r}^{j}=\rho_{k t}^{j}+C\left(d T_{r r}-d T_{k r}\right)=\sqrt{\left(X^{j}-X_{k}\right)^{2}+\left(Y^{j}-Y_{k}\right)^{2}}+C\left(d T_{r r}-d T_{k r}\right)
\end{aligned}
$$

As we calculate the position for dynamic receiver directly through three pseudolites, because of the error of positioning of the pseudolite itself, a small number of pseudolite positioning will lead to a large number of errors in positioning results. Moreover, the positioning accuracy is also related to the geometric arrangement of the three pseudolites, so the targeting range is limited. Therefore, in order to improve the positioning accuracy, a large number of pseudolite receiver can be set because of the low cost of ground pseudolite receiver set, and then through the centroid algorithm to solve the position.

Through a known moment of dynamic receiver position $\left(X_{k}, Y_{k}\right)$, it is possible to calculate the distance deviation $C\left(d T_{r r}-d T_{k r}\right)$ caused by the receiver deviation. So the real distance of the dynamic receiver k from the jth pseudolite is: $\rho_{k t}^{j}=P_{k}^{j}+\Delta \rho_{r}^{j}-C\left(d T_{r r}-d T_{k r}\right)$

In order to improve the positioning accuracy, a large number of pseudolite receivers can be set up and the position can be calculated by the centroid algorithm[2].

Specific algorithm process is as follows: 
a) There are m known position of the pseudolite receiver and they are divided into $C_{m}^{3}$ groups and each group has three nodes.

b) For one group, there are three nodes $A, B, C$ and their position are $\left(x_{a}, y_{a}\right),\left(x_{b}, y_{b}\right)$, and $\left(x_{c}\right.$, $\mathrm{y}_{\mathrm{c}}$ ). According to the pseudorange differential GPS method above, we can get the distance between an unknown dynamic receiver $M(x, y)$ from $A, B, C$ are $d_{a}, d_{b}$ and $d_{c}$ respectively. We have:

$$
\begin{aligned}
& \left(x-x_{a}\right)^{2}+\left(y-y_{a}\right)^{2}=d_{a}^{2}(8) \\
& \left(x-x_{b}\right)^{2}+\left(y-y_{b}\right)^{2}=d_{b}^{2}
\end{aligned}
$$

Simultaneous (8) and (9),we can get $\mathrm{M}_{\mathrm{ab} 1}\left(\mathrm{x}_{\mathrm{ab} 1}, \mathrm{y}_{\mathrm{ab} 1}\right)$ and $\mathrm{M}_{\mathrm{ab} 2}\left(\mathrm{x}_{\mathrm{ab} 2}, \mathrm{y}_{\mathrm{ab} 2}\right)$. If the distance form $\mathrm{M}_{\mathrm{ab} 1}$ to $\mathrm{C}$ is less than $\mathrm{M}_{\mathrm{ab} 2}$, then $\mathrm{M}_{\mathrm{ab}}\left(\mathrm{x}_{\mathrm{ab}}, \mathrm{y}_{\mathrm{ab}}\right)=\left(\mathrm{x}_{\mathrm{ab} 1}, \mathrm{y}_{\mathrm{ab} 1}\right)$, otherwise take $\mathrm{M}_{\mathrm{ab}}=\left(\mathrm{x}_{\mathrm{ab} 2}, \mathrm{y}_{\mathrm{ab} 2}\right)$. Similarly we can get $\mathrm{M}_{\mathrm{ac}}$ and $\mathrm{M}_{\mathrm{bc}}$.

Unlike conventional centroid algorithms, this method uses pseudoranges to calculate distances. Since the pseudolites method considers the distance deviation $C\left(d T_{r r}-d T_{k r}\right)$ caused by the receiver deviation to be approximately a same value, there exists an error. And the distance $\rho_{k t}^{j}$ from the closer pseudolite receiver is larger, and the error is bigger. So there are:

$$
x_{M 1}=\frac{d_{a b \mathrm{Xab}}+d_{a c} \mathrm{Xac}+d_{b c} \mathrm{Xbc}}{d_{a b}+d_{a c}+d_{b c}} \quad \text { (11) } \quad y_{M 1}=\frac{d_{a b} y_{\mathrm{ab}}+d_{a c} y_{\mathrm{ac}}+d_{b c} \mathrm{y}_{\mathrm{bc}}}{d_{a b}+d_{a c}+d_{b c}}
$$

Similarly, $C_{m}^{3}$ different $\mathrm{M}$ coordinate value can be obtained. Let $\mathrm{n}=C_{m}^{3}$, denoted as $\left\{D_{1}, D_{2}, D_{3}, \cdots, D_{n},\right\}$.

c) Then use the centroid location algorithm again for the set $\left\{D_{1}, D_{2}, D_{3}, \cdots, D_{n},\right\}$ and we get the final position node coordinates:

$$
\begin{aligned}
& x_{M}=\frac{d_{1} d_{2} d_{3} \mathrm{XD}_{1}+d_{1} d_{2} d_{4} \mathrm{XD}_{2}+\cdots+d_{n-2} d_{n-1} d_{n} \mathrm{XDn}_{\mathrm{Dn}}}{d_{1} d_{2} d_{3}+d_{1} d_{2} d_{4}+\cdots+d_{n-2} d_{n-1} d_{n} \mathrm{X \textrm {Dn }}} \\
& y_{M}=\frac{d_{1} d_{2} d_{3} y_{\mathrm{D} 1}+d_{1} d_{2} d_{4} y_{\mathrm{D} 2}+\cdots+d_{n-2} d_{n-1} d_{n} y_{\mathrm{Dn}}}{d_{1} d_{2} d_{3}+d_{1} d_{2} d_{4}+\cdots+d_{n-2} d_{n-1} d_{n} \mathrm{XDn}}
\end{aligned}
$$

$\mathrm{d}_{\mathrm{n}}$ is the distance between the pseudolite node and the unknown node.

\section{Error Analysis:}

Since the results of the centroid algorithm are directly related to the pseudorange from the pseudolite receivers, it is necessary to correct the pseudorange of pseudolite. Due to the large number of pseudolite receivers, the calculation of the pseudorange error correction is large. Therefore, in this paper, firstly, the parity correlation coefficient algorithm[3] is used to find the pseudolite receivers 
which have high correlation with the positioning results. Then, by using the phase smooth pseudorange differential measurement method, the pseudorange errors can be effectively reduced. From the parity algorithm, we have:

$$
p=P Z=P(H X+\varepsilon)=P H X+P \varepsilon=P \varepsilon=P_{1} \varepsilon_{1}+P_{2} \varepsilon_{2}+\cdots P_{n} \varepsilon_{n}
$$

$\mathrm{Pi}$ is the ith column of the $\mathrm{P}$ matrix, which is a vector that reflects the influence of $\varepsilon i$ to the parity vector $\mathrm{p}$. In this model, $\mathrm{p}$ is the coordinates of the final positioning result. $\varepsilon \mathrm{i}$ is the distance di corrected by differential GPS method between the pseudolite receiver and the unknown node.

The correlation of $\mathrm{p}$ and $\mathrm{Pi}$ is expressed by the minimum value of sum of the squares of the difference between $\mathrm{p}$ and $\mathrm{Pi}$ :

$$
Q(a, b)=\frac{1}{m} \sum_{j=1}^{m}\left(p_{j}-a-b^{*} P_{j i}\right)^{2}
$$

By taking the appropriate values for $\mathrm{a}$ and $\mathrm{b}$ to make $\mathrm{Q}$ minimum, and the smaller Qi is, the more relevant it is between $\mathrm{p}$ and $\mathrm{Pi}$. The partial derivative of $\mathrm{a}$ and $\mathrm{b}$ :

$$
\begin{aligned}
& \left\{\begin{array} { c } 
{ \frac { \partial Q } { \partial a } = - \frac { 2 } { m } \sum _ { j = 1 } ^ { m } ( p _ { j } - a - b P _ { j i } ) = 0 } \\
{ \frac { \partial Q } { \partial b } = - \frac { 2 } { m } \sum _ { j = 1 } ^ { m } ( p _ { j } - a ) P _ { j i } - b P _ { j i } { } ^ { 2 } ) = 0 }
\end{array} \quad \left\{\begin{array} { c } 
{ a = \overline { p } - b P _ { i } } \\
{ \quad \text { (17)Solution: } }
\end{array} \quad \left\{\begin{array}{c}
m=\sum_{j=1}^{m}(P j i-\bar{P})(p j-\bar{p}) / \sum_{j=1}^{m}(P j i-\bar{P})^{2}
\end{array}\right.\right.\right. \\
& \text { So: } \quad Q i=\frac{1}{n} \sum_{j=1}^{m}\left(p_{j}-\bar{p}\right)^{2}\left(1-\rho_{P p p}^{2}\right) \quad \text { (19)where } \rho_{P_{P p}}=\sum_{j=1}^{m}\left(P_{j i}-\bar{P}\right)\left(p_{j}-\bar{p}\right) /\left[\left(\sum_{j=1}^{m}\left(P_{j i}-\bar{P}\right)^{2}\right)\left(\sum_{j=1}^{m}(p j-\bar{p})^{2}\right)\right]^{\frac{1}{2}}
\end{aligned}
$$

$\rho^{P_{i p}}$ is the degree of correlation between the final location coordinate and the distance di between the pseudolite receiver and the unknown node. The larger the value is, the more relevant it is. Since p is a function of $\varepsilon$, it is stochastic. We think pi and Pij obey the normal distribution and the confidence a is given. When ${ }^{\rho_{P p}>t_{a}} / \sqrt{t_{a}^{2}+(n-2)}$, $\mathrm{P}$ was significantly correlated with $\mathrm{Pi}$ and the further error correction is required. ${ }^{t=\sqrt{\rho_{P_{p}}(n-2) /\left(1-\rho_{\left.P_{p}\right)}^{2}\right)}}$ obeys a t-distribution with $\mathrm{m}-2$ degrees of freedom.

This paper used phase smooth pseudorange differential measurements method to correct those pseudoranges from the significantly correlating satellites .

In addition to the pseudorange, the GPS receiver also provides carrier phase observations. The carrier phase observation is two orders of magnitude higher than the pseudorange observation accuracy. What's more, for the pseudolite system whose range is small, the GPS phase measurement noise can be approximately ignored. So we can use phase smooth pseudorange differential measurement[4] to improve accuracy.

Suppose pseudorange and phase observation equation are:

$$
\rho=P+C d \tau+V_{1}
$$

$$
\lambda(\varphi+N)=P+C d \tau+V_{2}
$$


Where $\rho$ is the pseudorange after correction; $d \tau$ is the distance deviation equals to $C\left(d T_{r r}-d T_{k r}\right)$; $\varphi$ is the observed phase fraction; $\mathrm{N}$ is the overall phase ambiguity; $\lambda_{\text {is }}$ the wavelength; $P$ is the $\mathrm{u}$ true distance from user to the pseudolite; V1, V2 are the measured noise for the receiver.

We take the difference between the phase observations at $\mathrm{t} 1$ and $\mathrm{t} 2$ is:

$\delta \rho\left(t_{2}, t_{1}\right)=\lambda\left(\varphi\left(t_{2}\right)-\varphi\left(t_{1}\right)\right)=P\left(t_{2}\right)-P\left(t_{1}\right)+C\left(d \tau\left(t_{2}\right)-d \tau\left(t_{1}\right)\right)+V_{2}$

It can be found that the ambiguity is eliminated. Because the base station and the user machine are not far away (within $100 \mathrm{~km}$ ) and GPS phase measurement noise level is millimeters, the visual V2 $\approx 0$ related to pseudorange observation. The pseudorange observation at time t2 is: $\rho\left(t_{2}\right)=P\left(t_{2}\right)+C d \tau\left(t_{2}\right)+V_{1}$

Substituting: $\rho\left(t_{2}\right)=P\left(t_{1}\right)+C d \tau\left(t_{1}\right)+\delta \rho\left(t_{2}, t_{1}\right)+V_{1}$

Considering the differential pseudorange observations of the noise mean is 0 , the differential pseudorange observations at $\mathrm{t} 1$ can be measured by the amount of phase change of the difference between the pseudorange between $\mathrm{t} 1$ and $\mathrm{t} 2$ :

$\rho\left(t_{1}\right)=\rho\left(t_{2}\right)-\delta \rho\left(t_{2}, t_{1}\right)$

As can be seen from the above equation, the pseudorange value at $\mathrm{t} 1$ can be calculated from the phase difference at different time. Suppose there are k ephemeris observations $\rho(\mathrm{t} 1), \rho(\mathrm{t} 2), \ldots, \rho(\mathrm{tk})$.Using the phase observation, we can obtain the phase differences from the t1 to tk and we can find pseudorange observations

at t1: $\left\{\begin{array}{c}\rho\left(t_{1}\right)=\rho\left(t_{1}\right) \\ \rho\left(t_{1}\right)=\rho\left(t_{2}\right)-\delta \rho\left(t_{2}, t_{1}\right) \\ \vdots \\ \rho\left(t_{1}\right)=\rho\left(t_{k}\right)-\delta \rho\left(t_{k}, t_{1}\right)\end{array}\right.$

So we have $\overline{\rho\left(t_{1}\right)}=\frac{1}{k} \sum \rho\left(t_{1}\right)(28)$

Then we can obtain the other value:

$\overline{\rho\left(t_{1}\right)}=\overline{\rho\left(t_{k}\right)}+\delta \rho\left(t_{1}, t_{k}\right)$

\section{Experimental simulation:}

40 pseudolites receivers and 10 carrier receivers with random different positions were selected in the $100 \times 100$ area. Suppose pseudolite receiver output pseudorange follows $(0,1)$ normal distribution. In 40 pseudolite receivers, we selected the receiver near the center as the base station. 10 times of output 
results are selected for phase smoothing pseudo-range differential measurement correction.

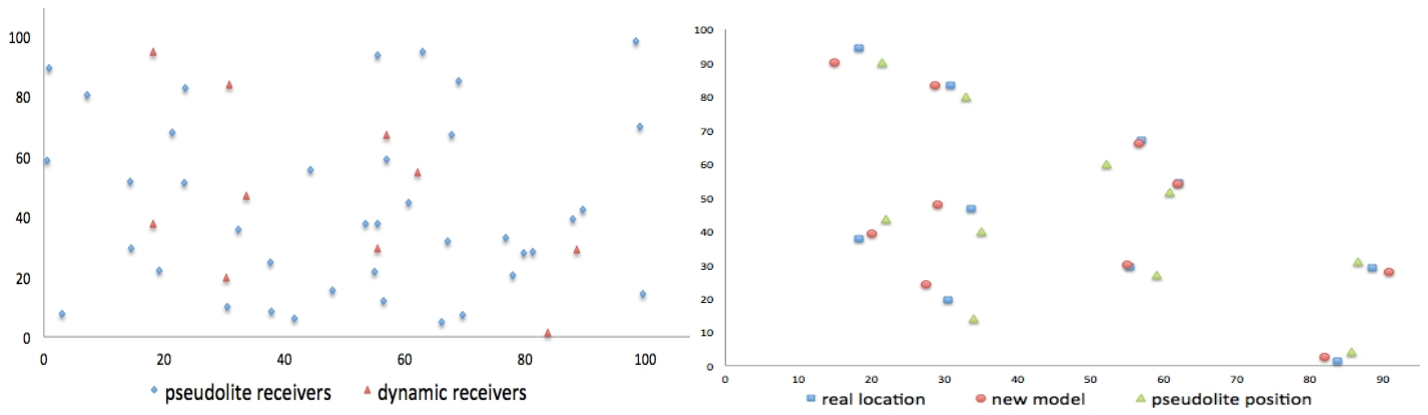

It can be seen that the improved model has a higher accuracy and the closer the receiver is to the receiver, the more accurate the positioning result is. Therefore, in practical applications, it need to carefully select the base receiver position.

\section{Acknowledgements:}

This work was financially supported by the Research Innovation Fund for College Students of Beijing University of Posts and Telecommunications.

\section{References:}

[1] Yuan Haiyi. GPS Navigation System Rapid Positioning Mthod Research[D]. Shenyang ligong university. 2014

[2] GAO Lei, ZHENG Xiang-quan, ZHANG Hong. A Node-Localization Algorithm for Wireless Sensor Network Based on Trilateration and Centroid Algorith[N].

JOURNAL OF CHONGQING INSTITUTE OF TECHNOLOGY. 2009,23(7)

[3] Zhang Liang. Research on GPS Navigation and Location Algorithm[D]. Northwestern Polytechnical University. 2007

[4] Li Heyuan. The Theory and Practice of Getting and Updating the Road Information of Vehicle[D]. Changan University. 2006 